\title{
Asymmetric dimethylarginine - a prognostic marker for transplant outcome?
}

\author{
Janghee Woo ${ }^{1,2}$ and H. Joachim Deeg ${ }^{1,2}$ \\ ${ }^{1}$ Fred Hutchinson Cancer Research Center and 'University of Washington School of Medicine, Seattle, WA, USA \\ E-mail: H.JOACHIM DEEG - jdeeg@fredhutch.org
}

doi:10.3324/haematol.2018.212191

$\mathrm{R}$ esults of allogeneic hematopoietic cell transplantation (HCT) have improved progressively over the past two decades. ${ }^{1}$ However, disease relapse, graftversus-host disease (GvHD), and various other causes of non-relapse mortality continue to be hurdles to greater success. These challenges have been tackled from various angles, both clinically and in the laboratory. One focus of research has been on the role of endothelial cells in the pathophysiology and manifestations of GvHD, sinusoidal obstruction syndrome, diffuse alveolar hemorrhage, and transplant-associated microangiopathy. There is a substantial body of literature on the effects of pro-inflammatory cytokines, derived from endothelial cells or other cellular compartments, on the interactions between donor-derived cells and host tissues and organs. ${ }^{2,3}$

In this issue of Haematologica, Radujkovic and colleagues examine a potential role of asymmetric dimethylarginine (ADMA), an endogenous compound derived from endothelial cells, as a pre-transplant marker for post-transplant complications. ${ }^{4}$ For this purpose, they studied data from 938 patients transplanted at two German centers who had serum samples collected within 4 weeks before transplantation and that were available for determination of ADMA levels. The results of their analysis indicate that higher levels of ADMA before HCT were associated with an increased risk of non-relapse mortality within the first year after transplantation. There was no association with relapse or GvHD. However, overall survival and progression-free survival during the first year after transplantation were negatively affected by higher pre-HCT levels of ADMA. Higher AMDA levels were also associated with shortened overall survival, shortened progression-free survival, and a higher incidence of non-relapse mortality within 1 year after the onset of acute GvHD. As ADMA is an endogenous molecule, the authors conclude that their findings underscore the importance of endothelial cell function for posttransplant outcome.

ADMA occurs naturally as a metabolic byproduct of protein modification processes in human cells, as first described by Vallance et al. in 1992..$^{5}$ One important function is its interference with L-arginine - via nitric oxide (NO) synthase ${ }^{6}$ - in the production of $\mathrm{NO}$, a molecule wellknown to be involved in endothelial function. The presence of elevated ADMA levels has, therefore, broad implications, including interference with vasodilation, facilitation of atherogenesis, insulin resistance, the development of autoimmune disorders, and rejection of kidney allografts, among others. ${ }^{7-10}$ It should not, therefore, be surprising that ADMA plays a significant role in disorders such as cardiovascular disease, diabetes mellitus, certain forms of renal disease, and in erectile dysfunction. ${ }^{6}$ ADMA levels increase substantially in response to native or oxidized low density lipoprotein cholesterol, thereby further inhibiting NO production. Studies in animal models have indicated that
ADMA increases at a time when vascular disease may not be clinically evident.

These data suggest that ADMA, originating from endothelial cells, may also directly affect endothelial function, which is relevant to transplant outcomes as reported by the authors. Reduced NO levels will alter tissue perfusion, resulting in organ dysfunction and, conceivably, greater susceptibility of lungs, intestinal tract, liver or kidneys to the effects of HCT conditioning regimens and the cytokines released as a consequence of donor/host interactions. ${ }^{2}$ As ADMA has also been suggested to be a potential biomarker for insulin resistance, ${ }^{10}$ it might be involved in altered blood glucose regulation, a problem for many patients after HCT. Furthermore, $\mathrm{NO}$ is a potent inhibitor of platelet aggregation and adhesion, as well as leukocyte adhesion, and thereby reduces the risk of thrombotic events. Thus, reduction of $\mathrm{NO}$ production in the presence of elevated levels of ADMA might facilitate the formation of microthrombi and contribute to microvascular dysfunction. ${ }^{11}$ The reference of the authors to post-transplant microangiopathy is, therefore, relevant, and it is unfortunate that no data on specific causes of death, in particular no histological data, were available.

Clearly, this paper raises many questions. As the authors note, they present an observational study, which does not allow any cause-and-effect relationship to be determined, and they remain undecided in their discussion. For example, it is puzzling that nitrate levels were elevated along with ADMA prior to transplantation, as were, incidentally, thrombomodulin levels, which were also associated with increased non-relapse mortality in a previous report. ${ }^{3}$ Since ADMA levels were analyzed in pre-HCT samples, a logical assumption would be that pre-HCT events, such as exposure to chemotherapy or infectious agents or, possibly, genetic factors, were responsible for raised levels of ADMA. There are data showing that a polymorphism in dimethylarginine dimethylaminohydrolase 2 (DDAH2), the enzyme that promotes ADMA metabolism, is associated with elevated ADMA levels, ${ }^{12}$ and it is tempting to speculate that this polymorphism might affect post-HCT nonrelapse mortality. Limited studies on polymorphism of the NO synthase gene by the authors failed to show a correlation with outcome, and such a polymorphism (or mutation) would not by itself explain elevated ADMA levels.

It is of interest to see that the authors provide outcome data, not only relative to the day of HCT, but also relative to the onset of GvHD, considering the possibility that the development of GvHD might have affected the subsequent course in an ADMA-dependent way. However, there was no significant impact of ADMA levels on post-GvHD outcome, relapse, overall survival, or progression-free survival, and the results were not different from those given in relation to the transplant date. What might one have expected?

The authors had reported previously that patients with 
(steroid-refractory) GvHD were those with elevated preHCT angiopoietin 2 levels. ${ }^{3}$ While the overall picture in that setting is more complex, are there potential interactions between elevated ADMA and angiopoietin 2 levels? Or are both elevated because of the same insult? It would have been of interest to see (in the present study) pre-transplant angiopoietin 2 levels and whether there was a correlation with ADMA concentrations.

Additional studies will be necessary to further dissect interactions between ADMA and other molecules, to follow ADMA levels longitudinally (for example, do levels change with the development of GvHD?), and to determine whether the time of onset of GvHD might have an impact (there was a wide time span during which GvHD developed). Furthermore, while a global comparison of highintensity and reduced-intensity conditioning regimens is provided, and early and late disease stages were considered, it would have been interesting to have had more specific data on the conditioning regimens and, for example, treatment of diseases prior to transplantation. This is not to diminish the authors' accomplishments; however, some of these studies will be necessary in order to provide a mechanistic explanation for the data.

In conclusion, the data must be interpreted with caution. This is particularly important when considering possible interventions. As suggested by the authors, high doses of citrulline may be useful to raise $\mathrm{L}$ arginine levels, counterbalancing the effect of ADMA. Could phosphodiesterase-5inhibitors (such as sildenafil) be useful to force the induction of NO synthase? Additional work should generate interesting data.

\section{References}

1. Gooley TA, Chien JW, Pergam SA, et al. Reduced mortality after allogeneic hematopoietic-cell transplantation. $N$ Eng J Med. 2010;363(22):2091-2101.

2. Antin JH, Ferrara JLM. Cytokine dysregulation and acute graft-versushost disease. Blood. 1992;80:2964-2968.

3. Luft T, Dietrich S, Falk C, et al. Steroid-refractory GVHD: T-cell attack within a vulnerable endothelial system. Blood. 2011;118(6):1685-1692.

4. Radujkovic A, Dai H, Kordelas L, et al. Asymmetric dimethylarginine serum levels are associated with early mortality after allogeneic stem cell transplantation. Haematologica. 2019;104(4):827-834.

5. Vallance P, Leone A, Calver A, Collier J, Moncada S. Accumulation of an endogenous inhibitor of nitric oxide synthesis in chronic renal failure. Lancet. 1992;339(8793):572-575.

6. Forstermann U, Sessa WC. Nitric oxide synthases: regulation and function. Eur Heart J. 2012;33(7):829-837, 837a-837d.

7. Matsuguma K, Ueda S, Yamagishi S, et al. Molecular mechanism for elevation of asymmetric dimethylarginine and its role for hypertension in chronic kidney disease. J Am Soc Nephrol. 2006;17(8):2176-2183.

8. Franceschelli S, Ferrone A, Pesce M, Riccioni G, Speranza L. Biological functional relevance of asymmetric dimethylarginine (ADMA) in cardiovascular disease. Int J Mol Sci. 2013;14(12):24412-24421.

9. Sibal L, Agarwal SC, Home PD, Boger RH. The role of asymmetric dimethylarginine (ADMA) in endothelial dysfunction and cardiovascular disease. Curr Cardiol Rev. 2010;6(2):82-90.

10. Lee W, Lee HJ, Jang HB, et al. Asymmetric dimethylarginine (ADMA) is identified as a potential biomarker of insulin resistance in skeletal muscle. Sci Rep. 2018;8(1):2133.

11. Jodele S, Dandoy CE, Myers KC, et al. New approaches in the diagnosis, pathophysiology, and treatment of pediatric hematopoietic stem cell transplantation-associated thrombotic microangiopathy. Transfus Apher Sci. 2016;54(2):181-190.

12. Xuan C, Xu LQ, Tian OW, et al. Dimethylarginine dimethylaminohydrolase 2 (DDAH 2) gene polymorphism, asymmetric dimethylarginine (ADMA) concentrations, and risk of coronary artery disease: a case-control study. Sci Rep. 2016;6:33934. 